\title{
Desarrollo de la sagitta en juveniles y adultos de Odontesthes argentinensis (Valenciennes, 1835) y O. bonariensis (Valenciennes, 1835) de la provincia de Buenos Aires, Argentina (Teleostei: Atheriniformes)
}

\author{
Development of the sagitta in young and adults of Odontesthes argentinensis \\ (Valenciennes, 1835) and Odontesthes bonariensis (Valenciennes, 1835) from Buenos \\ Aires province, Argentina (Teleostei: Atheriniformes)
}

ANDREA D. TOMBARI*, ALEJANDRA V. VOLPEDO \& DINORAH D. ECHEVERRÍA
Laboratorio de Vertebrados, Departamento de Biodiversidad y Biología Experimental, Facultad de Ciencias Exactas y Naturales, Ciudad Universitaria Pabellón 2, (1428), Buenos Aires, Argentina;
*e-mail: tombari@bg.fcen.uba.ar

\begin{abstract}
RESUMEN
Se describió el desarrollo del otolito Sagitta de juveniles y adultos de Odontesthes argentinensis procedentes de Punta Rasa $\left(36^{\circ} 22^{\prime} \mathrm{S}\right)$ y Miramar (38 $\left.16^{\circ} \mathrm{S}\right)$ y de Odontesthes bonariensis de la Laguna de Chascomús $\left(35^{\circ} 36^{\prime}\right.$ $\mathrm{S})$. Se estudió, la morfología de las sagittae de ambas especies, analizando cuatro grupos morfológicos para $O$. argentinensis (I: $75-120 \mathrm{~mm}$, II: 130-220 mm, III: 230-270 y IV: 280-320 mm) y tres grupos morfológicos para O. bonariensis (I: $80-120 \mathrm{~mm}$, II: $130-220$ y III: $230-320 \mathrm{~mm}$ ). Las características morfológicas comunes a la sagitta, de ambas especies, en los grupos estudiados son: presencia de sulcus, depresión areal dorsal en la cara medial, cara lateral con estrías y la presencia de un punto culminante en el borde dorsal. En la sagitta de $O$. argentinensis, la depresión areal dorsal se inicia ligada a la cauda, característica que permitió distinguir claramente entre otolitos de juveniles de ambas especies. A partir del estado adulto, la depresión areal dorsal se halla separada de la cauda en las dos especies y el análisis estadístico evidenció un crecimiento discontinuo. Se hallaron diferencias morfológicas entre pares de sagitta de un mismo individuo en cuanto a la forma del ostium, presencia de cisura, rostro y punto culminante, aunque no se halló significancia morfométrica.
\end{abstract}

Palabras clave: morfología, sagitta, Odontesthes, Atherinopsidae.

\begin{abstract}
The development of the otolith sagitta was described in young and adults of two silverside species. Odontesthes argentinensis was collected from Punta Rasa ( $36^{\circ} 22^{\prime}$ S) and Miramar ( $38^{\circ} 16^{\prime} \mathrm{S}$ ) and, Odontesthes bonariensis collected from Laguna de Chascomús $\left(35^{\circ} 36^{\prime} \mathrm{S}\right)$. Sagitta morphology was studied in both species, analyzing four morphological groups of $O$. argentinensis (I: 75-120 mm, II: 130-220 mm, III: 230-270 mm and IV: 280$320 \mathrm{~mm}$ ) and three morphological groups of $O$. bonariensis (I: $80-120 \mathrm{~mm}$, II: 130-220 mm and III: 230-320 $\mathrm{mm}$ ). The morphological features of the sagitta shared by both species are: sulcus present, dorsal areal depression on the medial face, lateral face with striations and the presence of the culminant point on the dorsal margin. The dorsal areal depression of $O$. argentinensis begins in contact with the cauda. This feature easily identifies the sagittae of young in both species. The dorsal areal depression is separated from the cauda in adults of both species, and the statistical analysis showed a discontinuous growth. Morphological differences were found between pairs of sagittae of the same specimen relating to the shape of the ostium, presence of an anterior excisure, rostrum and the culminant point, but no significant morphometric difference was found.
\end{abstract}

Key words: morphology, sagitta, Odontesthes, Atherinopsidae.

\section{INTRODUCCIÓN}

Los pejerreyes pertenecen a un taxon bien representado en Argentina con 10 especies: Odontesthes argentinensis, O. smitti (Lahille, 1929), O. incisa (Jenyns, 1841), O. platensis
(Berg, 1895) y O. nigricans (Richardson, 1848) para el ambiente marino; y O. bonariensis, $O$. hatcheri (Eigenmann, 1909), O. perugiae (Evermann \& Kendall, 1906), O. retropinnis (de Buen, 1953) у O. humensis (de Buen, 1953) para ambientes continentales (Dyer 1997). 
Odontesthes argentinensis y $O$. bonariensis son las especies de pejerreyes de mayor relevancia de la República Argentina, por su importancia comercial y recreativa, ya que alcanzan tallas promedio de $370 \mathrm{~mm}$ y $400 \mathrm{~mm}$, respectivamente (Cousseau \& Perrota 2000, Grosman et al. 2001).

La distribución original de $O$. argentinensis se

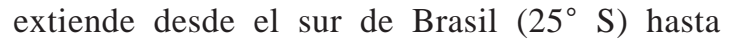
Rawson $\left(43^{\circ} 2^{\prime} \mathrm{S}\right)$, Chubut, Argentina (García 1987) y $O$. bonariensis se halla frecuentemente en aguas continentales. Esta última especie tiene una amplia distribución debido a que fue introducida en distintos cuerpos de agua y actualmente se la encuentra desde el Lago Titicaca $\left(16^{\circ} 20^{\prime} \mathrm{S}\right)$, Bolivia, sur de Brasil y hasta aguas patagónicas de la Argentina (Dyer 1998).

La taxonomía del género Odontesthes ha sido reorganizada por García (1987) y Dyer (1997). Estos autores utilizaron características osteológicas, morfológicas y enzimáticas para el establecimiento de las relaciones filogenéticas del grupo.

Ecológicamente, ambas especies son componentes importantes de la dieta de numerosos ictiófagos, principalmente $O$. argentinensis (Ringuelet 1943, Fitch \& Brownell 1971, Ringuelet et al. 1980). Los estudios de ecología trófica se basan en la identificación de las presas por el reconocimiento de estructuras específicas, como huesos y otolitos (Hecht 1987, Gosztonyi \& Kuba 1996, Volpedo \& Echeverría 2000). La morfología de la sagitta de adultos de cuatro especies de pejerreyes de Argentina fue establecida por Tombari et al. (2000). Brown \& Fuentes (2001) hallaron que la forma de los otolitos de larvas de Odontesthes bonariensis difiere de la de los juveniles y adultos. Se ha comprobado que en la ontogenia de Micropogonias furnieri (Demarest, 1923), la sagitta experimenta importantes variaciones morfológicas (Volpedo \& Echeverría 1999).

De acuerdo con lo arriba mencionado, se propone caracterizar el desarrollo de las sagittae de juveniles y adultos de $O$. argentinensis y $O$. bonariensis con el fin de aplicarlas a estudios de ecología trófica.

\section{MATERIALES Y MÉTODOS}

Los ejemplares de Odontesthes argentinensis fueron capturados en las áreas costeras marinas de Punta Rasa $\left(36^{\circ} 22^{\prime}\right.$ S) y Miramar $\left(38^{\circ} 16^{\prime}\right.$ $\mathrm{S})$, y los de $O$. bonariensis de la laguna de Chascomús $\left(35^{\circ} 6^{\prime} \mathrm{S}\right)$, provincia de Buenos Aires, República Argentina (Fig. 1). Los peces fueron colectados en el período comprendido entre los meses de mayo de 1998 hasta octubre de 1999, con una batería de redes de diferente malla operadas perpendicularmente a la costa para $O$. argentinensis, y con un conjunto de espineles y una serie de mediomundos para $O$. bonariensis.

Los especímenes fueron congelados a una temperatura de $-18{ }^{\circ} \mathrm{C}$ hasta el momento de ser examinados en el laboratorio. Los ejemplares fueron identificados según las claves propuestas por García (1987) y Dyer (1997). Se registró la longitud total (LT) de los peces en $\mathrm{mm}$ y se extrajeron las sagittae de las cápsulas óticas para su observación y medición. Luego se ilustraron las caras medial y lateral, y la vista dorsal de las sagittae derechas. Las características morfológicas y morfométricas fueron registradas con un microscopio estereoscópico con error menor que $0,1 \mathrm{~mm}$. La terminología utilizada para la descripción morfológica es la propuesta por Volpedo \& Echeverría (2000). Para las caras de las sagittae se utilizaron los términos medial y lateral en reemplazo de interna y externa respectivamente.

Se analizaron 66 sagittae de $O$. argentinensis y 96 sagittae de $O$. bonariensis con la finalidad de evaluar el desarrollo de las mismas a través del incremento en tamaño del pez.

Sobre la base de las características de importancia taxonómica se establecieron cuatro grupos morfológicos de las sagittae para $O$. argentinensis y tres para $O$. bonariensis en relación con la longitud total (LT) del pez para cada especie (Tabla 1). De acuerdo con la observación preliminar de la muestra de otolitos se consideraron los siguientes caracteres que surgen o se modifican morfológicamente durante el desarrollo de la sagitta, tales como: (a) forma general; (b) forma de los bordes; (c) presencia del punto culminante, que se define como el punto más elevado del borde dorsal (Mollo 1981); (d) topografía de la cara medial y lateral; (e) conformación del sulcus; (f) posición relativa de la depresión areal dorsal y (g) rostro. 


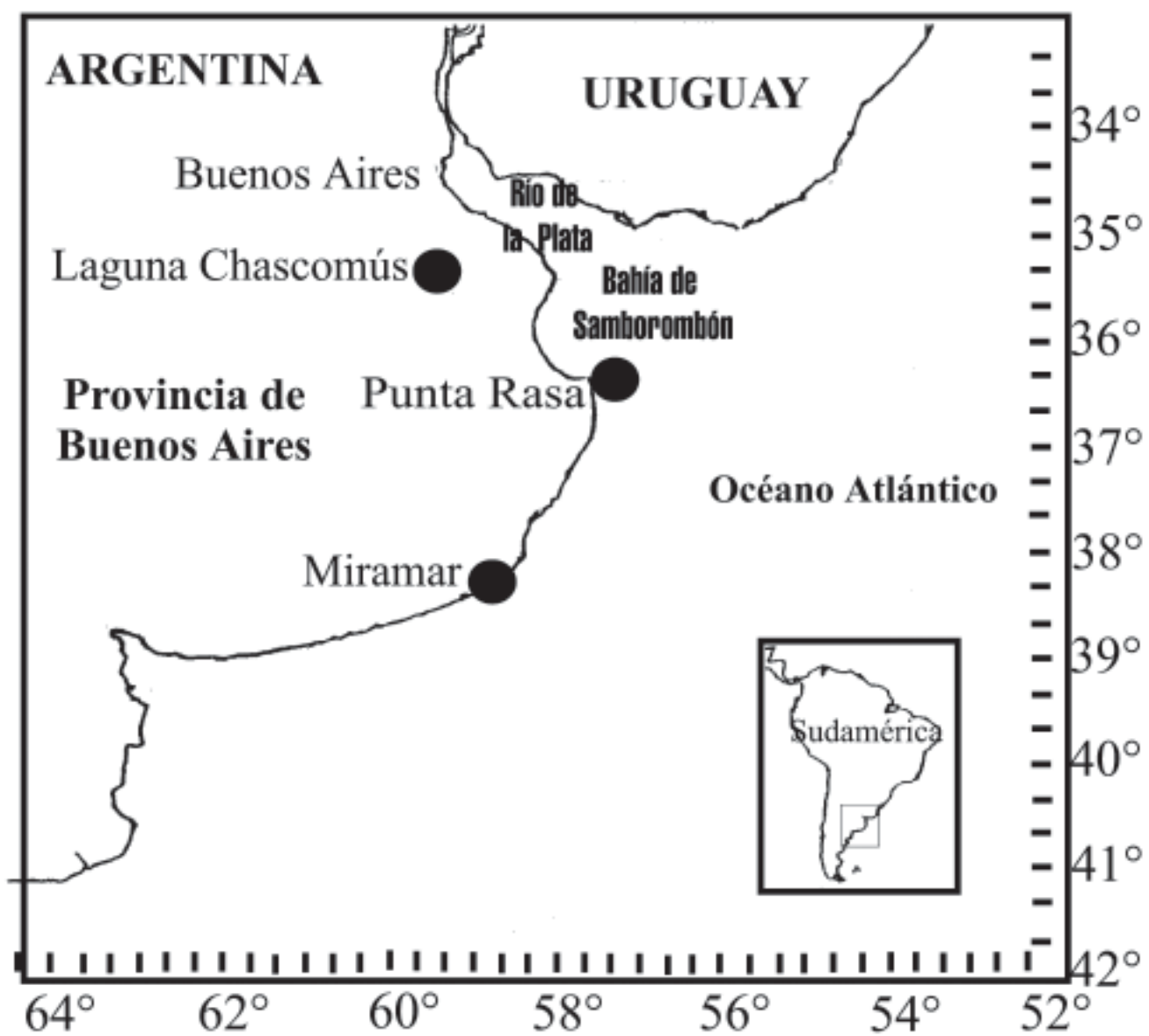

Fig. 1: Ubicación de las localidades de procedencia de las muestras.

Location of sampling localities.

TABLA 1

Grupos morfológicos de la sagitta de $O$. argentinensis y $O$. bonariensis. LT = longitud total del pez; $\mathrm{n}=$ número de muestras

Sagitta morphological groups of $O$. argentinensis and $O$. bonariensis. $\mathrm{LT}=$ total length; $\mathrm{n}=$ total number of sagittae samples

\begin{tabular}{ccccccc}
\hline & \multicolumn{2}{c}{ O. argentinensis } & & \multicolumn{3}{c}{ O. bonariensis } \\
\cline { 2 - 3 } \cline { 5 - 6 } Grupo & LT $(\mathrm{mm})$ & $\mathrm{n}$ & & Grupo & LT $(\mathrm{mm})$ & $\mathrm{n}$ \\
\hline I & $75-120$ & 19 & I & $80-120$ & 3 \\
II & $130-220$ & 35 & II & $130-220$ & 86 \\
III & $230-270$ & 9 & III & $230-320$ & 7 \\
IV & $280-320$ & 3 & & & \\
\hline
\end{tabular}

Se registraron los siguientes caracteres: largo máximo de la sagitta (LO), ancho máximo de la sagitta $(\mathrm{AO})$, largo máximo del ostium (LOS), ancho máximo del ostium (AOS), largo máximo de la cauda (LC) y ancho máximo de la cauda (AC), expresados en $\mathrm{mm}$. Para ambas especies se verificó la existencia de la igualdad morfométrica entre la sagitta derecha e izquierda mediante la prueba $t$ de Student (Sokal \& Rohlf 1995, Zar 1999).

Para cada especie y en cada localidad se establecieron las siguientes relaciones entre los caracteres morfométricos de las sagittae y la longitud total del pez: LT vs. LO, LT vs. AO, LT vs. LC, LT vs. AC, LT vs. LOS y LT vs. AOS, y se calcularon los coeficientes de la regresión lineal y el coeficiente de determinación. Las ecuaciones de regresión obtenidas para cada localidad fueron 
comparadas a través de análisis de covarianza (ANCOVA).

Los valores de los caracteres morfométricos de las sagittae de cada grupo fueron comparados aplicando un análisis de varianza (ANOVA) (Sokal \& Rohlf 1995), utilizando como covariable la LT y aplicando un test de comparaciones múltiples a posteriori (Tukey) para diseños no balanceados con una significancia del cinco por ciento. Previamente a la aplicación del ANOVA se comprobaron los supuestos de normalidad y homocedacea por los tests de Kolmogorov-Smirnov (Lillifords) y Levene (Sokal \& Rohlf 1995, Zar 1999) respectivamente.

El programa estadístico utilizado en el análisis de los datos fue STATISTICA 5.1 (Statsoft, 1999). La colección de sagittae se halla depositada en el Laboratorio de Vertebrados bajo la denominación C.O.L.V.FCEN-UBA.

\section{RESULTADOS}

\section{Sagitta de O. argentinensis}

En el grupo I la forma es oblonga, los bordes ventral (Ve) y dorsal (Do) son irregulares y convexos (Fig. 2). El punto culminante (P) aparece como un esbozo. Las caras medial $(\mathrm{Cm})$ y lateral $(\mathrm{Cl})$ poseen estrías radiales, siendo convexa la primera y la última plana. En el sulcus (S), el ostium (Os) puede encontrarse dividido por la cisura (E) o ser esta poco pronunciada. La depresión areal dorsal (De) se encuentra en contacto estrecho con la cauda (Ca) y el rostro (R) puede ser largo o corto (Fig. 2-GI).

En el grupo II la forma es oblonga, los bordes son convexos e irregulares, principalmente el dorsal, donde se observa el punto culminante. Las caras medial y lateral con estrías dispuestas irregularmente sobre los bordes, siendo convexa la primera y la última cóncava. El ostium puede presentar una pequeña cisura. La depresión areal dorsal se encuentra en contacto parcial o total con la cauda y el rostro es evidente (Fig. 2-GII).

En el grupo III la forma es oblonga pero de aspecto alargado debido a que los bordes van perdiendo su convexidad. Se observa el punto culminante desarrollado en el borde dorsal. Las caras medial y lateral presentan escasas estrías radiales, siendo convexa la primera y la última cóncava. En el ostium se puede hallar una pequeña cisura. La depresión areal dorsal se encuentra separada y paralela a la cauda. El rostro, si existe, está poco desarrollado (Fig. 2GIII).

En el grupo IV la forma es oblonga, pero de aspecto alargado debido a que los bordes se encuentran casi paralelos a la cauda, estos son de aspecto regular y presentan el punto culminante en el borde dorsal. La cara medial es lisa y la lateral tiene escasas estrías irregulares, siendo convexa la primera y la última cóncava. El ostium posee una cisura marcada. La depresión areal dorsal se encuentra separada y paralela a la cauda. El rostro es evidente (Fig. 2-GIV).

\section{Sagitta de O. bonariensis}

En el grupo I la forma es oblonga, ambos bordes son irregulares, principalmente el dorsal por la presencia del punto culminante (Fig. 3). Las caras medial y lateral presentan estrías radiales en los bordes, siendo la primera levemente convexa y la segunda plana. El sulcus es de aspecto granular y el ostium puede presentar una muy pequeña cisura. La depresión areal dorsal se encuentra paralela a la cauda pero no contacta con ella. El rostro es alargado (Fig. 3-GI).

En el grupo II la forma es oblonga, ambos bordes son irregulares, principalmente el dorsal por la presencia del punto culminante. La cara medial presenta escasas estrías radiales en los bordes y la cara lateral con numerosas estrías radiales, siendo convexa la primera y la última cóncava. El sulcus es de aspecto granular y el ostium puede presentar cisura. La depresión areal dorsal se encuentra paralela a la cauda pero no en contacto con ella. El rostro se mantiene (Fig. 3-GII).

En el grupo III la forma es oblonga. Los bordes son irregulares, principalmente el dorsal por la presencia del punto culminante. La cara medial con escasas estrías radiales y la cara lateral con estrías de disposición irregular. El sulcus es de aspecto granular y el ostium puede tener una cisura evidente. La depresión areal dorsal se encuentra paralela a la cauda pero no contacta con ella. Con rostro evidente (Fig. 3-GIII). 

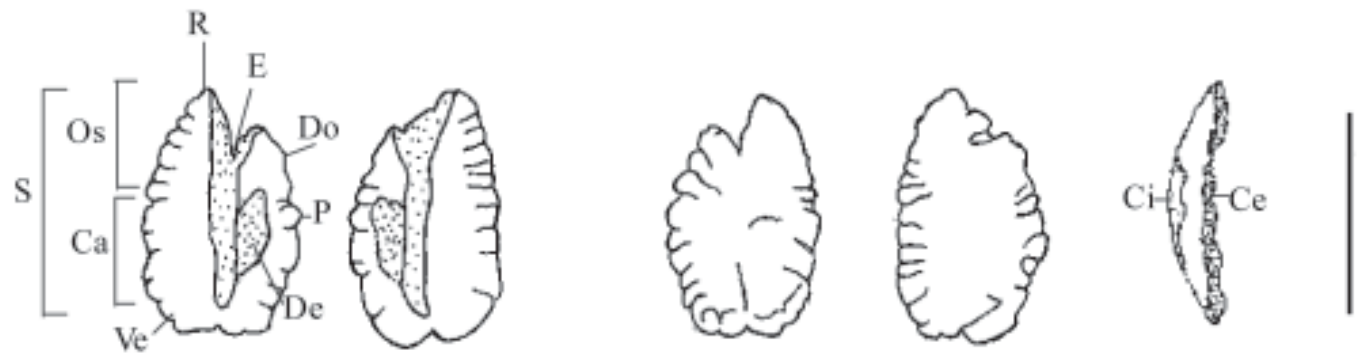

GI
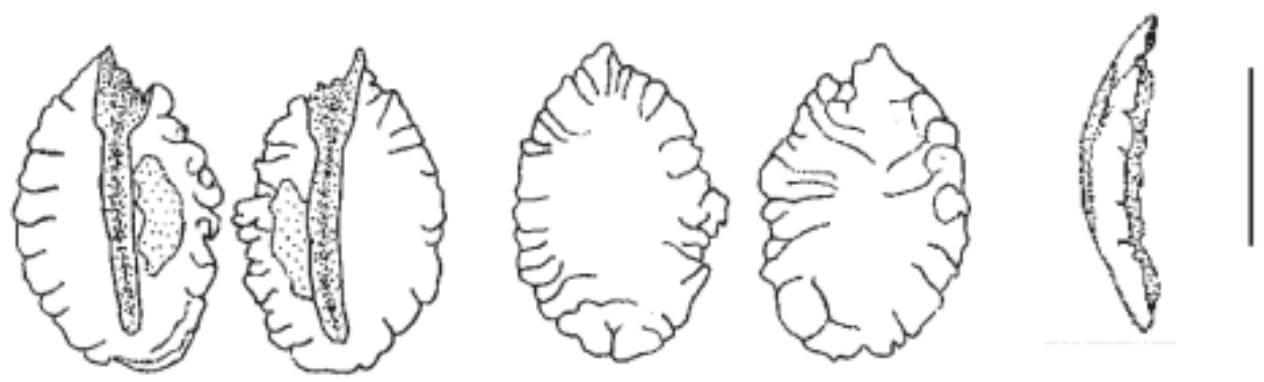

GII
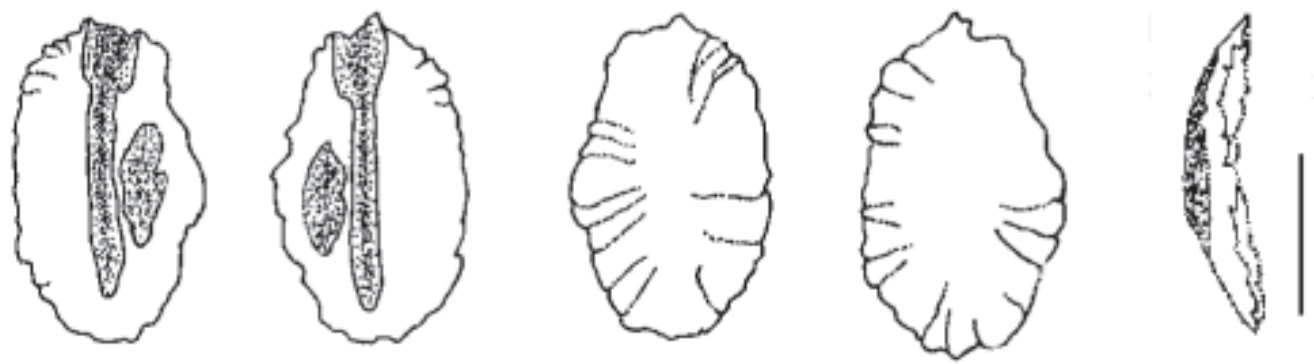

GIII
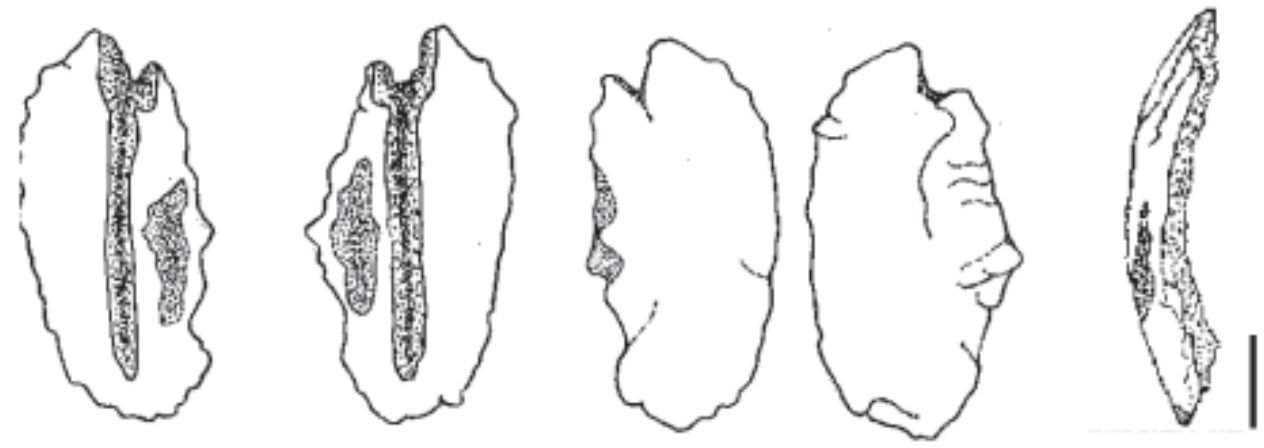

GIV

Fig. 2: Morfología de las sagittae de juveniles y adultos de $O$. argetinensis. De izquierda a derecha: cara medial de sagitta derecha, cara medial de sagitta izquierda, cara lateral de sagitta derecha, cara lateral de sagitta izquierda, vista dorsal derecha. GI = Grupo I $(75-120 \mathrm{~mm})$, GII = Grupo II $(130-$ $220 \mathrm{~mm})$, GIII = Grupo III $(230-270 \mathrm{~mm})$, GIV = Grupo IV $(280-320 \mathrm{~mm})$. Escala $=3 \mathrm{~mm}$.

Sagittae morphology of young and adult Odontesthes argentinensis. From left to right: medial face of right sagitta, medial face of left sagitta, lateral face of right sagitta, lateral face of left sagitta, right dorsal view. GI = Group I (75-120 mm), GII $=$ Group II $(130-220 \mathrm{~mm})$, GIII = Group III $(230-270 \mathrm{~mm})$, GIV = Group IV $(280-320 \mathrm{~mm})$. Scale $=3 \mathrm{~mm}$. 

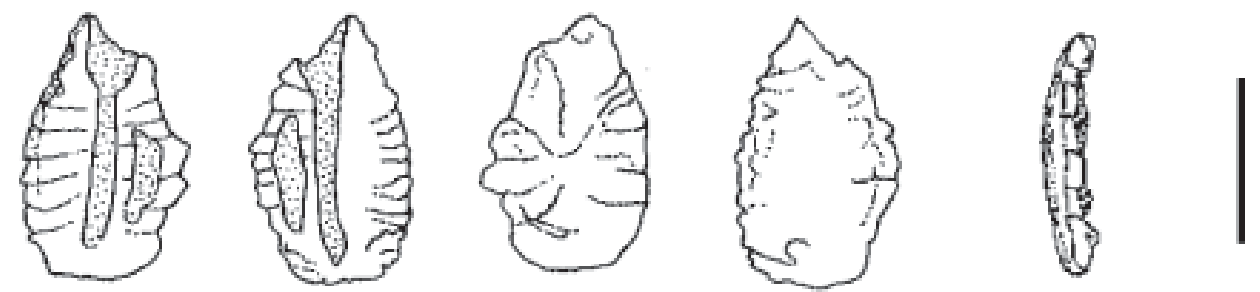

GI
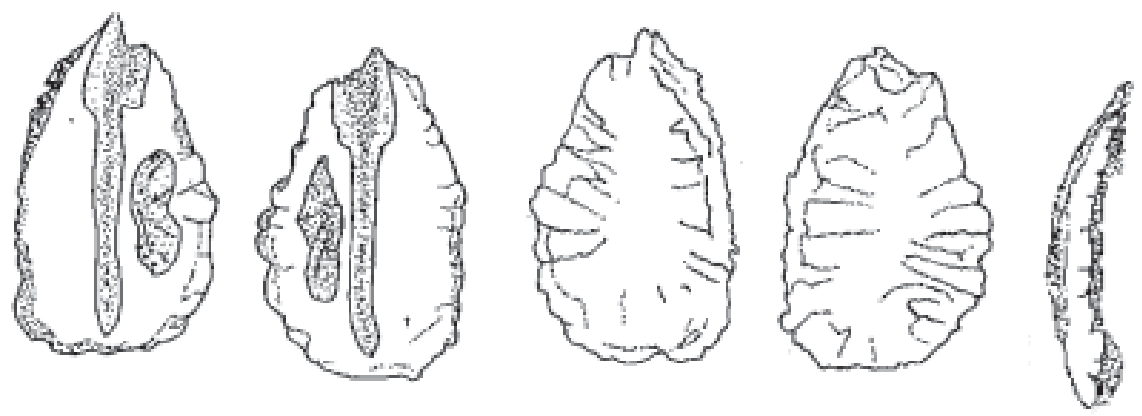

GII
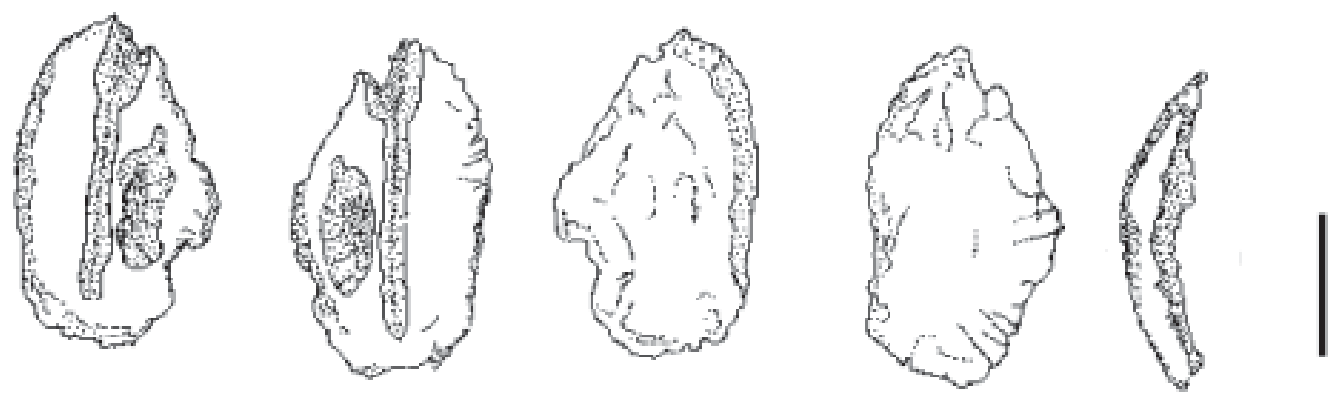

GIII

Fig. 3: Morfología de las sagittae de juveniles y adultos de O. bonariensis. De izquierda a derecha: cara medial de sagitta derecha, cara medial de sagitta izquierda, cara lateral de sagitta derecha, cara medial de sagitta izquierda, vista dorsal derecha. GI = Grupo I (80-120 mm), GII = Grupo II (130$220 \mathrm{~mm})$, GIII = Grupo III $(230-320 \mathrm{~mm})$. Escala $=3 \mathrm{~mm}$.

Sagittae morphology of young and adult specimens of Odontesthes bonariensis. From left to right: medial face of right sagitta, medial face of left sagitta, lateral face of right sagitta, lateral face of left sagitta, right dorsal view. GI = Group I $(80-120 \mathrm{~mm})$, GII $=$ Group II $(130-220 \mathrm{~mm})$, GIII $=$ Group III $(230-320 \mathrm{~mm})$. Scale $=3 \mathrm{~mm}$.

En ambas especies las sagittae izquierda y derecha de cada ejemplar resultaron morfométricamente iguales (Tabla 2).

Los parámetros de la regresión y el coeficiente de determinación entre los caracteres morfométricos de la sagitta de $O$. argentinensis y la longitud total del pez se presentan en la Tabla 3, resultando todas las regresiones significativas. Los coeficientes de determinación de LO, AO y LC son altos para las sagittae de $O$. argentinensis de Punta Rasa y Miramar, no evidenciándose diferencias significativas entre las sagittae de los peces de cada localidad bonaerense, por lo que ambas muestras pertenecerían a la misma población estadística, lo que permitió reunirlas en un mismo grupo.

Las relaciones morfométricas entre los caracteres de la sagitta y el LT de $O$. argentinensis de los diferentes grupos, presentaron diferencias significativas entre los grupos (Tabla 4). El test de Tukey para cada carácter morfométrico de la sagitta analizado, 
evidenció diferencias significativas entre el LT y LO, AO y LC. Para el grupo I se diferencia de los restantes en el AOS, el grupo II no presenta diferencias significativas en el AC y el LOS respecto al grupo III. El grupo III no presenta diferencias significativas respecto del grupo IV en el LOS (Tabla 5).

En O. bonariensis, los caracteres morfométricos de la sagitta mostraron diferencias significativas entre los grupos, excepto para el AOS (Tabla 4). La prueba de Tukey para cada carácter morfométrico de la sagitta analizado, evidenció diferencias significativas entre los tres grupos (Tabla 6).

\section{DISCUSIÓN}

El estudio de la morfología de los otolitos de $O$. argentinensis y $O$ bonariensis puso en evidencia características propias de cada especie y en común con otros pejerreyes. Se halló un patrón común de la sagitta de juveniles y adultos referidos a la morfología de las caras medial y lateral. El patrón morfológico de las sagittae de adultos coincide con la descripción efectuada por Tombari et al. (2000) para estas dos especies, así como con la descripción de $O$. bonariensis realizada por Mollo (1973). Ambas tienen en común la forma oblonga, el ostium en forma de copa, la cauda alargada, extendida por el centro del otolito y una depresión areal dorsal dispuesta paralelamente a la cauda. Los caracteres comunes que comparten con otras especies como $O$. hatcheri y $O$. smitti son: en la cara medial, la división del sulcus en ostium y cauda, y la presencia de una depresión areal en la cara medial de la sagitta en posición dorsal y en la cara lateral las sagittae poseen estrías y pliegues (Tombari et al. 2000). Asimismo, estas carácterísticas fueron las observadas por Mollo (1974) en Odontesthes incisa y por Harvey et al. (2000) en Atherinops affinis (Ayres, 1860) y Atherinopsis californiensis (Girard, 1854), por lo que conforman un patrón morfológico que podría representar a la subfamilia Atherinopsinae.

TABLA 2

Valores morfométricos (medias \pm DE) de la sagitta y resultados de las pruebas t de Student para las especies $O$. argentinensis y $O$. bonariensis. $\mathrm{AC}=$ ancho máximo de la cauda; $\mathrm{AO}=$ ancho máximo de la sagitta; AOS = ancho máximo del ostium; DE = desvío estándar; LC = largo máximo de la cauda, LO = largo máximo de la sagitta; LOS = largo máximo del ostium

Morphmetric values (means \pm SD) and Student t-test results between sagittae of both species. AC = maximum width of cauda $; \mathrm{AO}=$ maximum width of sagitta; $\mathrm{AOS}=$ maximum width of ostium; $\mathrm{DE}=$ standard deviation; $\mathrm{LC}=$ maximum length of cauda; $\mathrm{LO}=$ maximum length of sagitta; LOS = maximum length of ostium

\begin{tabular}{|c|c|c|c|c|}
\hline $\begin{array}{l}\text { Caracteres morfométricos } \\
\text { de la sagitta }\end{array}$ & Otolito derecho (mm) & Otolito izquierdo (mm) & *Valor de t & Valor de $\mathrm{P}$ \\
\hline \multicolumn{5}{|l|}{ O. argentinensis } \\
\hline LO & $3,415 \pm 1,137$ & $3,405 \pm 1,446$ & 0,053 & $0,957 \mathrm{~ns}$ \\
\hline $\mathrm{AO}$ & $2,108 \pm 0,675$ & $2,100 \pm 0,663$ & 0,065 & $0,948 \mathrm{~ns}$ \\
\hline $\mathrm{LC}$ & $2,212 \pm 0,808$ & $2,215 \pm 0,809$ & $-0,021$ & $0,982 \mathrm{~ns}$ \\
\hline $\mathrm{AC}$ & $0,293 \pm 0,106$ & $0,281 \pm 0,067$ & 0,780 & $0,436 \mathrm{~ns}$ \\
\hline LOS & $0,786 \pm 0,259$ & $0,796 \pm 0,258$ & $-0,235$ & $0,814 \mathrm{~ns}$ \\
\hline AOS & $0,496 \pm 0,160$ & $0,512 \pm 0,166$ & $-0,560$ & $0,576 \mathrm{~ns}$ \\
\hline \multicolumn{5}{|l|}{ O. bonariensis } \\
\hline LO & $3,584 \pm 0,967$ & $3,588 \pm 0,966$ & 0,0273 & $0,978 \mathrm{~ns}$ \\
\hline $\mathrm{AO}$ & $2,236 \pm 0,459$ & $2,236 \pm 0,472$ & 3,138 & $0,999 \mathrm{~ns}$ \\
\hline LC & $2,188 \pm 0,568$ & $2,184 \pm 0,563$ & 0,040 & $0,968 \mathrm{~ns}$ \\
\hline $\mathrm{AC}$ & $0,302 \pm 0,045$ & $0,303 \pm 0,044$ & 0,169 & $0,865 \mathrm{~ns}$ \\
\hline LOS & $0,907 \pm 0,326$ & $0,926 \pm 0,302$ & $-0,411$ & $0,681 \mathrm{~ns}$ \\
\hline AOS & $0,538 \pm 0,132$ & $0,539 \pm 0,114$ & $-0,030$ & $0,976 \mathrm{~ns}$ \\
\hline
\end{tabular}

(*) $\mathrm{t}_{130}$ para $O$. argentinensis y $\mathrm{t}_{190}$ para $O$. bonariensis 
Parámetros de la regresión lineal funcional de los caracteres morfométricos de la sagitta de $O$.

argentinensis en relación con la longitud total del pez y resultados del ANCOVA entre los parámetros de regresión de las dos localidades estudiadas: $\mathrm{a}=$ ordenada al origen, $\mathrm{b}=$ pendiente, $\mathrm{r}^{2}$ = coeficiente de determinación; $\mathrm{AC}=$ ancho máximo de la cauda; $\mathrm{AO}=$ ancho máximo de la sagitta; $\mathrm{AOS}$ = ancho máximo del ostium; $\mathrm{LC}=$ largo máximo de la cauda; $\mathrm{LO}=$ largo máximo de la sagitta; LOS = largo máximo del ostium; $\mathrm{LT}=$ longitud total del pez;

$$
(* *)=\mathrm{P}<0,0001 ;(\mathrm{ns})=\mathrm{P}>0,05
$$

\begin{tabular}{|c|c|c|c|c|c|c|}
\hline \multirow[t]{2}{*}{ Relaciones } & \multirow[t]{2}{*}{ Localidad } & \multicolumn{3}{|c|}{ Parámetros de la regresión } & \multicolumn{2}{|c|}{ ANCOVA } \\
\hline & & a & $\mathrm{b}$ & $\mathrm{r}^{2}$ & $\mathrm{~F}_{1,34}$ & $\mathrm{~F}_{1,30}$ \\
\hline \multirow[t]{2}{*}{ LT versus LO } & Punta Rasa & 0,797 & 0,015 & 0,893 & $118,70 * *$ & $192,97 \mathrm{~ns}$ \\
\hline & Miramar & 0,029 & 0,019 & 0,990 & & \\
\hline \multirow[t]{2}{*}{ LT versus AO } & Punta Rasa & 0,737 & 0,008 & 0,887 & $110,69 * *$ & $63,947 \mathrm{~ns}$ \\
\hline & Miramar & $-0,030$ & 0,012 & 0,978 & & \\
\hline \multirow[t]{2}{*}{ LT versus LC } & Punta Rasa & 0,503 & 0,010 & 0,874 & $63,947 * *$ & $179,48 \mathrm{~ns}$ \\
\hline & Miramar & $-0,060$ & 0,012 & 0,990 & & \\
\hline \multirow[t]{2}{*}{ LT versus AC } & Punta Rasa & $-0,165$ & 0,002 & 0,658 & $23,003 * *$ & $40,312 \mathrm{~ns}$ \\
\hline & Miramar & 0,111 & 0,001 & 0,874 & & \\
\hline \multirow[t]{2}{*}{ LT versus LOS } & Punta Rasa & 0,237 & 0,003 & 0,695 & $28,133 * *$ & $52,658 \mathrm{~ns}$ \\
\hline & Miramar & $-0,016$ & 0,005 & 0,970 & & \\
\hline \multirow[t]{2}{*}{ LT versus AOS } & Punta Rasa & 0,303 & 0,001 & 0,408 & $6,023 * *$ & $22,019 \mathrm{~ns}$ \\
\hline & Miramar & 0,040 & 0,002 & 0,958 & & \\
\hline
\end{tabular}

Cabe destacar que en la sagitta de $O$. argentinensis, la depresión areal dorsal se inicia ligada a la cauda, característica que permite distinguir claramente entre los otolitos de juveniles de $O$. argentinensis y $O$. bonariensis. A partir del estado adulto, en estas especies, la depresión areal dorsal se halla independiente de la cauda. Ambas especies presentan gran variabilidad en el rostro y en la cisura, no solo durante el desarrollo de la sagitta, sino también entre las sagittae derecha e izquierda de un mismo individuo, independientemente de la longitud del pez.

El rostro en $O$. bonariensis se evidencia desde la etapa larval según lo observado en la imagen de Brown \& Fuentes (2001). Hemos observado que el rostro se encuentra muy desarrollado en los juveniles de menor talla y disminuye con el aumento de la misma. Las sagittae de $O$. argentiniensis presentan rostro evidente en los juveniles de menor talla estudiados, haciéndose menos marcado en los individuos de mayor talla.

En los primeros estadios juveniles de $O$. argentinensis, la cisura es evidente, mientras que en $O$. bonariensis esta característica se observa en todas las tallas. Esta estructura puede estar presente en la sagitta izquierda y/o en la derecha de ambas especies, por lo que podría sugerirse que el crecimiento de las sagittae es diferente para ambos otolitos de un mismo individuo aunque compartan el patrón general, lo cual es sustentado por el estudio morfométrico efectuado. 
TABLA 4

Resultados del ANOVA entre los caracteres morfométricos de la sagitta y la longitud total del pez de los distintos grupos para ambas especies. $\mathrm{AC}=$ ancho máximo de la cauda; $\mathrm{AO}=$ ancho máximo de la sagitta; $\mathrm{AOS}=$ ancho máximo del ostium; $\mathrm{LC}=$ largo máximo de la cauda; $\mathrm{LO}=$ largo máximo de la sagitta; LOS = largo máximo del ostium; LT = longitud total del pez. Las diferencias estadísticas significativas y altamente significativas se indican con $(*)$ y $(* *)$, respectivamente

\begin{tabular}{|c|c|c|c|c|}
\hline \multirow[t]{2}{*}{ Carácter morfométrico } & \multicolumn{2}{|c|}{ O. argentinensis } & \multicolumn{2}{|c|}{ O. bonariensis } \\
\hline & Valor de $F_{3,65}$ & Valor de $\mathrm{P}$ & Valor de $F_{2,95}$ & Valor de $\mathrm{P}$ \\
\hline $\mathrm{O}$ & 4,167 & $0,009 *$ & 6,148 & $0,003^{*}$ \\
\hline $\mathrm{AO}$ & 11,032 & $<0,0001 * *$ & 12,208 & $<0,0001 * *$ \\
\hline $\mathrm{C}$ & 3,430 & $0,022 *$ & 5,390 & $0,006^{*}$ \\
\hline $\mathrm{AC}$ & 8,651 & $<0,0001 * *$ & 8,027 & $0,0006^{*}$ \\
\hline OS & 2,978 & $0,038 *$ & 16,841 & $<0,0001 * *$ \\
\hline AOS & 2,786 & $0,048^{*}$ & 2,630 & 0,077 \\
\hline
\end{tabular}

\section{TABLA 5}

Resultados de pruebas de Tukey entre los grupos de $O$. argentinensis para la relación LT versus AC: I: $75-120 \mathrm{~mm}$, II: $130-220 \mathrm{~mm}$, III: 230-270 mm y IV: 280-320 mm; AC = ancho máximo de la cauda; $\mathrm{AO}=$ ancho máximo de la sagitta; $\mathrm{AOS}=$ ancho máximo del ostium; $\mathrm{LC}=$ largo máximo de la cauda; LO = largo máximo de la sagitta; LOS = largo máximo del ostium; LT = longitud total del pez. Las diferencias estadísticas no significativas, altamente significativas y no significativas se indican con $(*),(* *),(\mathrm{ns})$, respectivamente

Tukey test results within groups of $O$. argentinensis for the relationship LT verus AC: I: $75-120 \mathrm{~mm}$, II: $130-220 \mathrm{~mm}$, III: 230-270 mm and IV: 280-320 mm; AC = maximum width of cauda; $\mathrm{AO}=$ maximum width of sagitta; $\mathrm{AOS}=$ maximum width of ostium; $\mathrm{LC}=$ maximum length of cauda; $\mathrm{LO}=$ maximum length of sagitta; $\mathrm{LOS}=$ maximum length of ostium; LT $=$ total length of fish. Statistically, highly statistically, and statistically nonsignificant differences are indicated with $(*)$, $(* *),(\mathrm{ns})$, respectively

\begin{tabular}{|c|c|c|c|c|c|c|c|}
\hline $\mathrm{LT}$ versus $\mathrm{LO}$ & I & II & III & $\mathrm{LT}$ versus $\mathrm{AO}$ & I & II & III \\
\hline II & $<0,0001 * *$ & & & II & $<0,0001 * *$ & & \\
\hline III & $<0,0001 * *$ & $<0,0001 * *$ & & III & $<0,0001^{* *}$ & $0,003 *$ & \\
\hline IV & $<0,0001 * *$ & $<0,0001 * *$ & $<0,0001 * *$ & IV & $<0,0001 * *$ & $<0,0001 * *$ & $0,001^{*}$ \\
\hline LT versus LC & $\mathrm{I}$ & II & III & LT versus AC & I & II & III \\
\hline II & $<0,0001 * *$ & & & II & $<0,0001 * *$ & & \\
\hline III & $<0,0001 * *$ & $0,0003 *$ & & III & $<0,0001 * *$ & $0,425 \mathrm{~ns}$ & \\
\hline IV & $<0,0001 * *$ & $<0,0001 * *$ & $<0,0001 * *$ & IV & $<0,0001 * *$ & $<0,0001 * *$ & $<0,0001 * *$ \\
\hline LT versus LOS & I & II & III & LT versus AOS & I & II & III \\
\hline II & $<0,0001 * *$ & & & II & $<0,0001^{* *}$ & & \\
\hline III & $<0,0001 * *$ & $0,084 \mathrm{~ns}$ & & III & $<0,0001 * *$ & $0,391 \mathrm{~ns}$ & \\
\hline IV & $<0,0001 * *$ & $0,0009^{*}$ & $0,077 \mathrm{~ns}$ & IV & $<0,0001 * *$ & $0,132 \mathrm{~ns}$ & $0,649 \mathrm{~ns}$ \\
\hline
\end{tabular}




\section{TABLA 6}

Resultados de las pruebas de Tukey entre los grupos de $O$. bonariensis para las distintas relaciones morfométricas: I: $80-120 \mathrm{~mm}$, II: 130-220 mm, III: $230-320 \mathrm{~mm} ; \mathrm{AC}=$ ancho máximo de la cauda; $\mathrm{AO}=$ ancho máximo de la sagitta; LC = largo máximo de la cauda; LO = largo máximo de la sagitta; LOS = largo máximo del ostium; LT = longitud total del pez; Las diferencias estadísticas significativas y altamente significativas se indican con (*) y $(* *)$, respectivamente

Results from Tukey tests between groups of $O$. bonariensis for the different morphometric relationships: I: 80-120 mm, II: $130-220 \mathrm{~mm}$, III: $230-320 \mathrm{~mm}$; AC = maximum width of cauda; $\mathrm{AO}=$ maximum width of sagitta; $\mathrm{LC}=$ maximum length of cauda; $\mathrm{LO}=$ maximum length of sagitta; $\mathrm{LOS}=$ maximum length of ostium; $\mathrm{LT}=$ total length of fish. Statistically and highly statistically significant differences are indicated with $(*)$ and $(* *)$, respectively

\begin{tabular}{lcccc}
\hline LT versus LO & \multicolumn{3}{c}{ LT versus AO } \\
\hline & \multicolumn{1}{c}{ I } & II & I & II \\
\cline { 2 - 5 } II & $<0,0001 * *$ & & $<0,0001^{* *}$ & \\
III & $<0,0001 * *$ & $<0,0001 * *$ & $<0,0001^{* *}$ & $<0,0001^{* *}$
\end{tabular}

LT versus LOS

I II

II $\quad 0,002 *$

III $<0,0001 * *<0,0001 * *$

\begin{tabular}{lllll}
\hline LT versus LC & \multicolumn{3}{c}{ LT versus AC } \\
\hline \multicolumn{1}{c}{ I } & II & \multicolumn{1}{c}{ I } & II \\
\cline { 2 - 5 } & II & $<0,0001^{* *}$ & & $0,0002^{*}$ \\
III & $<0,0001^{* *}$ & $<0,0001^{* *}$ & $<0,0001^{* *}$ & $<0,0001 * *$ \\
\hline
\end{tabular}

El desarrollo del rostro y la cisura de los juveniles corresponde al patrón ecomorfológico de los otolitos de peces neríticos nadadores, propuesto por Volpedo \& Echeverría (2003), tanto para $O$. argentinensis como para $O$. bonariensis.

Otras características que también tienen relevancia taxonómica y que hacen a la distinción entre estas especies son: los bordes irregulares, principalmente el dorsal por la presencia del punto culminante, que se encuentra más desarrollado en $O$. bonariensis que en $O$. argentinensis. Además, el sulcus de
$O$. bonariensis posee un aspecto granular que se mantiene durante el desarrollo de la sagitta y adquiere un ancho constante a partir de los 240 $\mathrm{mm}$, que coinciden con la talla de la madurez sexual que fuera observada por Grosman et al. (2001).

Las variaciones en la forma general del sulcus y del punto culminante podrían estar asociados al modo en que se deposita el carbonato de calcio durante el desarrollo de la sagitta, de modo similar al que fuera observado por Volpedo \& Echeverría (1999) en las sagittae de Micropogonias furnieri.

La variabilidad morfológica observada durante el crecimiento podría estar asociada a factores biológicos (madurez sexual, crecimiento) y ecológicos (dinámica del cuerpo de agua, características físico químicas del agua, entre otras) que escapan al objetivo de este trabajo. La sagitta de $O$. bonariensis alcanza la forma definitiva, con el punto culminante prominente, rostro y cisura marcados a partir de los $230 \mathrm{~mm}$, talla que está en el rango de la estimada por Grosman et al. (2001) para la madurez sexual. En $O$. argentinensis la forma definitiva se adquiere a partir de los $280 \mathrm{~mm}$, con la depresión areal separada completamente de la cauda. Indicios de esta característica se registran en tallas desde $230 \mathrm{~mm}$, las cuales podrían asociarse al momento de madurez sexual de $O$. argentinensis. Esto se sustenta en las observaciones de 12 ejemplares de diferente sexo de nuestra muestra.

Tejedor (2001) afirma que $O$. argentinensis y $O$. bonariensis son especies sumamente difíciles de diferenciar por su morfología semejante e interfértiles entre sí. Esto podría explicar la variabilidad morfológica que hemos observado dentro del mismo par de otolitos de un mismo ejemplar y además de la compleja sistemática del grupo valorada por Dyer (1998).

\section{AGRADECIMIENTOS}

Al Dr. Brian Dyer por los valiosos comentarios y al Sr. Rolvider O. Galimany y amigos de la pesca deportiva por donarnos los ejemplares de $O$. argentinensis y $O$. bonariensis. 


\section{LITERATURA CITADA}

BROWN DR \& CM FUENTES (2001) Validación de los depósitos diarios de incrementos en otolitos de larvas del pejerrey bonaerense Odontesthes bonariensis (Pisces, Atherinidae). Instituto Nacional de Investigación y Desarrollo Pesquero. Informe Técnico 69: 1-13.

COUSSEAU MB \& RG PERROTA (2000) Peces marinos de Argentina, biología, distribución, pesca. Instituto Nacional de Investigación y Desarrollo Pesquero. Mar del Plata, Argentina. $167 \mathrm{pp}$

DYER B (1997) Phylogenetic revision of Atherinopsinae (Teleostei, Atherinopsidae), with comments on the systematics of the South American freshwater fish genus Basilichthys Girard. Miscelaneous Publications of the Museum of Zoology, University of Michigan (USA) 185: 1-64.

DYER B (1998) Phylogenetic systematics and historical biogeography of the neotropical silverside family Atherinopsidae (Teleostei: Atheriniformes). En: Malabarba LR, RE Reis, RP Vari, ZM Lucena \& CAS Lucena (eds) Phylogeny and classification of Neotropical fishes. Part 6 - Atherinomorpha: 519536. Edipucrs, Porto Alegre, Brasil.

FITCH JE \& RL BROWNELL (1971) Food habits of the franciscana Pontoporia blainvillei (Cetacea: Platanistidae) from South America. Bulletin Marine Science 21: 626-636.

GARCÍA ML (1987) Contribución al conocimiento sistemático y biológico de los Atherinidae del mar argentino. Tesis Doctoral, Universidad Nacional de La Plata, Facultad de Ciencias Exactas y Naturales y Museo, Mar del Plata, Argentina . 200 pp.

GOSZTONYI AE \& L KUBA (1996) Atlas de huesos craneales y de la cintura escapular de peces costeros patagónicos. Fundación Patagonia Natural. Plan de Manejo Integral de la Zona Costera Patagónica. Informe Técnico. Argentina. 4: $29 \mathrm{pp}$.

GROSMAN F, P SANZANO, G GONZÁLEZ, D AGÜERÍA \& S SERGUEÑA (2001) Ecología reproductiva, edad, crecimiento, condición y alimentación del pejerrey Odontesthes bonariensis en un ambiente del SO de la provincia de Buenos Aires, Argentina. Aquatic 12:1-24.

HARVEY JT, TR LOUGHLIN, MA PÉREZ \& DS OXMAN (2000) Relationship between fish size and otolith length for 63 species of fishes from the eastern north Pacific Ocean . Natioanl Oceanic \& Atmospheric Administration (US Department of
Commerce, NOAA), Technical Report National Marine Fisheries Service (NMFS) USA 150: 1-38.

HECHT T (1987) A guide to the otoliths of southern ocean fishes. South African Journal of Antarctic Research 17: $2-86$.

MOLLO SM (1973) Contribución al conocimiento del otolito sagitta de los Atherínidos argentinos. I . Basilichthys bonariensis (Pisces, Atherinomorpha). Physis (Argentina) B 32: 287-295.

MOLLO SM (1974) Contribución al conocimiento del otolito sagitta de los Atherínidos argentinos. II . Austrotherina incisa (Pisces, Atherinomorpha). Physis (Argentina) A 33: 505-509.

MOLLO SM (1981) Otolitos de peces de la Laguna Chascomús (Provincia de Buenos Aires). Análisis y consideraciones para su identificación en estudios tróficos. Limnobios 2: 253-263.

RINGUELET RA (1943) Piscicultura del pejerrey o atherinicultura. Agro (Argentina) 6: 1-162.

RINGUELET RA, R IRIART \& AH ESCALANTE (1980) Alimentación del pejerrey (Basilichthys bonariensis, Atherinidae) en laguna de Chascomús (Buenos Aires, Argentina). Relaciones ecológicas de complementación y eficiencia trófica del plancton. Limnobios 1: 447-460.

SOKAL RR \& FJ ROHLF (1995) Biometry. Third edition. W.H. Freeman and Company, New York, New York, USA. 887 pp.

TEJEDOR D (2001) El pejerrey como recurso genético. En: Grosman F (ed) Fundamentos biológicos, económicos y sociales para una correcta gestión de recurso pejerrey: 27-31. Editorial Astyanax, Azul, Buenos Aires, Argentina

TOMBARI AD, VOLPEDO AV \& DD ECHEVERRÍA (2000) Patrones morfológicos de la sagitta de pejerreyes de la ictiofauna argentina. Thalassas 16: $11-19$

VOLPEDO AV \& DD ECHEVERRÍA (1999) Morfología de los otolitos sagittae de juveniles y adultos de Micropogonias furnieri (Demarest, 1923)(Scianidae). Thalassas 15: 19-24.

VOLPEDO AV \& DD ECHEVERRÍA (2000) Catálogo y claves de otolitos para la identificación de peces del mar argentino. Editorial Dunken, Buenos Aires, Argentina. $88 \mathrm{pp}$

VOLPEDO AV \& DD ECHEVERRÍA (2003) Ecomorphological patterns of the sagitta in fish on the continental shelf off Argentine. Fisheries Research 60: 551-560.

ZAR JH (1999) Biostatistical analysis. Fourth edition. Prentice Hall, Englewood Cliffs, New Jersey, 663 pp. 